\title{
Mineraçãa
}

\section{Depressores para dolomita na flotação de willemita}

\section{Depressants for dolomites in willemite flotation}

\author{
Carlos Alberto Pereira \\ Dr. em Tecnologia Mineral, \\ Professor Efetivo do Departamento \\ de Engenharia de Minas da UFOP \\ pereira@demin.ufop.br

\section{Caroline Belisário Zorzal \\ Graduanda da Engenharia de Minas do Departamento de Engenharia \\ de Minas da UFOP \\ carolinezorzal@gmail.com \\ Lucas Carvalho Coelho \\ Mestrando do Curso de Engenharia \\ Mineral da UFOP, Engenheiro de Minas \\ da Fundação Gorceix \\ Icarvalhocoelho@yahoo.com.br}

\section{Michelly dos Santos Oliveira}

Dra. em Tecnologia Mineral,

Professora do Curso de Mineração

do IFMG - Campus Ouro Preto

o.michelly@ifmg.edu.br

\section{Resumo}

Essa investigação avaliou o efeito de depressores para dolomita na flotação de zinco, a fim de aumentar tanto a recuperação quanto a seletividade. Os reagentes estudados foram: uma emulsão de amina (Clariant Flotigan 2835-2L), óleo diesel e MIBIC, nas proporções 1,0; 0,16 e 0,4 como coletor e espumante; solução de sulfeto de sódio e barrilha como agente ativador e modificador de $\mathrm{pH}$, respectivamente; silicato de sódio como agente dispersante e como depressores: silicato de sódio, dextrina, dicromato de potássio, tanino, amido e carboximetilcelulose (CMC). As etapas de dispersão, sulfetização e flotação de willemita foram investigadas. Esse trabalho foi desenvolvido em escala de laboratório e apresentou, entre outros resultados, um bom desempenho da carboximetilcelulose e amido como depressores da ganga dolomítica na flotação do minério de zinco willemítico.

Palavras-chave: Flotação de willemita, depressores, dolomita.

\begin{abstract}
This research addressed the effects of depressants on willemite flotation, in order to increase both zinc recovery and selectivity. The reagents were: an amine emulsion (Clariant Flotigan 2835-2L), diesel oil and MIBIC in the proportions of 1.0, 0.16 and 0.4 as the collector and frother; a sodium sulfide and sodium carbonate solution as the activator and $\mathrm{pH}$ modifier, respectively; sodium silicate as the dispersant agent; and as the depressant: sodium silicate, dextrin, potassium dichromate, tannin, starch and carboxymethylcellulose (CMC). The steps of dispersion, sulfidization and willemite flotation were investigated. This work was conducted on laboratory scale and showed, among other findings, that a good performance could be obtained using carboxymethylcellulose and starch as a dolomitic gangue depressant in the flotation of willemite zinc ore.
\end{abstract}

Keywords: Willemite flotation, depressants, dolomite.

\section{Introdução}

Os principais minerais de zinco encontrados em depósitos brasileiros são willemita, esfalerita e hemomorfita. As reservas, em sua maioria, são de minerais de zinco oxidados, compostos de silicatos e carbonatos, friáveis, com alta porcentagem de finos e lamas, o que dificulta sua concentração.

O concentrador de Vazante - MG trata minérios de zinco do tipo oxidado (os principais minerais-minério são willemita, hemimorfita e smithsonita e os principais minerais de ganga são a dolomita, o quartzo e a hematita). O processo de beneficiamento consiste nas operações de britagem, moagem e flotação. A preparação desse material para a flotação envolve as etapas de dispersão dos sólidos, sulfetização, adição do coletor e dos agentes espumantes. A importância dos depósitos de minérios oxidados de zinco de Vazante chamou a atenção de pesquisadores bra- 
sileiros nas décadas de 70, 80 e primeira metade de 90. Muitos trabalhos, como os Salum et al. (1992), Borges (1993), Viana (1981), Viana e Peres (1982), Baltar (1980) e Galéry (1985), confirmaram a relevância da sulfetização e da dispersão.

Atualmente, na flotação do minério willemítico de Vazante, são usados os seguintes reagentes: sulfeto de sódio $1400 \mathrm{~g} / \mathrm{t}$ (produto do petróleo ativador), metilisobutilcarbinol (MIBIC) 50g/t (álcool, espumante), silicato de sódio $300 \mathrm{~g} / \mathrm{t}$ (dispersante) e dodecilamina $250 \mathrm{~g} / \mathrm{t}$ (coletor). Depressores não são empregados, porém a aplicação deles poderia melhorar a seletividade da flotação, já que esse minério de zinco se encontra associado a óxidos de ferro e carbonato de cálcio e magnésio, indesejáveis ao produto.

Segundo Salum et al. (1992), a depressão da ganga é requerida devido

\section{Materiais e métodos}

As amostras de willemita estudadas são provenientes da alimentação da flotação do concentrador da Votorantim Metais de Vazante e foram coletadas e enviadas pela própria empresa. Os trabalhos de laboratório foram realizados nas instalações do Departamento de Engenharia de Minas da UFOP.

A composição química das amostras, obtida por espectrometria de fluorescência de raios X e realizada pelo Laboratório à resposta similar dos minerais de zinco e aqueles de ganga na flotação catiônica. Ensaios de microflotação em amostras de calcita, apatita e dolomita "puras" foram executados por Luvizotto e Lima (2003), sendo testados vários reagentes para a depressão dos minerais calcita e dolomita, tais como amido de milho, silicato de sódio, fosfato de sódio e tartarato de sódio e potássio. $\mathrm{O}$ amido de milho mostrou-se o mais eficiente. Vale lembrar que o amido de milho também contribui na depressão de óxidos de ferro na flotação de minério de ferro e fosfato (Guimarães et al., 2005). Zheng e Smith (1997), na flotação de apatita, e Pereira (2004), na flotação de calamina não deslamada, demonstraram o bom desempenho da CMC na depressão de dolomita. Na flotação de calamina, Pereira e Peres (2005) estudaram a ação dos depressores amido de milho, carbo-

da Unidade de Vazante da Votorantim, está descrita na Tabela 1 e a distribuição granulométrica é apresentada na Figura 1. A análise granulométrica foi realizada pelo método do peneiramento combinado, com o desbaste em uma peneira de 400 mesh $(38 \mu \mathrm{m})$. Por meio da Figura 1, pode-se determinar o $\mathrm{d}_{80}$ do minério em $110 \mu \mathrm{m}$.

Os depressores testados foram silicato de sódio $(300 \mathrm{~g} / \mathrm{t})$, dextrina $(1200 \mathrm{~g} / \mathrm{t})$, dicromato de potássio $(40 \mathrm{~g} / \mathrm{t})$, tanino ximetilcelulose (CMC), silicato de sódio e dicromato de potássio para a depressão de dolomita e concluíram que CMC e silicato de sódio foram mais efetivos.

O presente trabalho tem como objetivo investigar o processo de flotação do minério oxidado de zinco da unidade da Votorantim Metais em Vazante, testando diversos reagentes depressores para os minerais de ganga, principalmente a dolomita. Grande parte da tecnologia desenvolvida para o beneficiamento de minérios de zinco é voltada para os minérios sulfetados. Por isso se faz necessário buscar melhorias no desempenho da concentração de minérios oxidados, almejando incrementar a recuperação metalúrgica do zinco e diminuir o consumo de reagentes, principalmente ácido sulfúrico, na metalurgia, em razão da presença do $\mathrm{Ca}$ e $\mathrm{Mg}$ no concentrado do minério.

\begin{tabular}{c|c|c|c|c|c|c|c|c}
\hline Amostra & $\mathbf{Z n} \%$ & $\mathrm{Fé} \%$ & $\mathrm{Al}_{2} \mathrm{O}_{3} \%$ & $\mathrm{CaO} \%$ & $\mathrm{MgO} \%$ & $\mathrm{SiO}_{2} \%$ & $\mathrm{~Pb} \%$ & $\mathrm{~S} \%$ \\
\hline A1 & 17.20 & 6.42 & 1.14 & 14.03 & 10.55 & 11.94 & 0.55 & 0.03 \\
\hline A2 & 17.31 & 6.42 & 1.13 & 14.28 & 11.03 & 12.27 & 0.56 & 0.03 \\
\hline A3 & 16.46 & 6.24 & 1.10 & 13.31 & 9.29 & 11.01 & 0.53 & 0.03 \\
\hline A4 & 17.20 & 6.36 & 1.14 & 14.20 & 10.96 & 12.10 & 0.55 & 0.03 \\
\hline A5 & 16.98 & 6.38 & 1.17 & 13.84 & 10.46 & 11.84 & 0.55 & 0.03 \\
\hline A6 & 17.36 & 6.53 & 1.17 & 14.25 & 11.28 & 12.71 & 0.55 & 0.03 \\
\hline Teor médio & 17.09 & 6.39 & 1.14 & 13.99 & 10.60 & 11.98 & 0.55 & 0.03 \\
\hline
\end{tabular}

$(100 \mathrm{~g} / \mathrm{t})$, amido $(1000 \mathrm{~g} / \mathrm{t})$ e carboximetilcelulose $(100 \mathrm{~g} / \mathrm{t})$. As concentrações foram predeterminadas a partir de revisão bibliográfica e testes preliminares. Foi testada, também, uma mistura de silicato de sódio e poliacrilato $(300 \mathrm{~g} / \mathrm{t})$. Foi empregado, como agente ativador e modificador de $\mathrm{pH}$, o sulfeto de sódio a uma dosagem de $700 \mathrm{~g} / \mathrm{t}$, sendo que a solução foi preparada a uma concentração de $4,9 \%(50 \%$ do sulfeto quimicamente ativo). Assim, o

Tabela 1

Análise química das amostras de willemita.

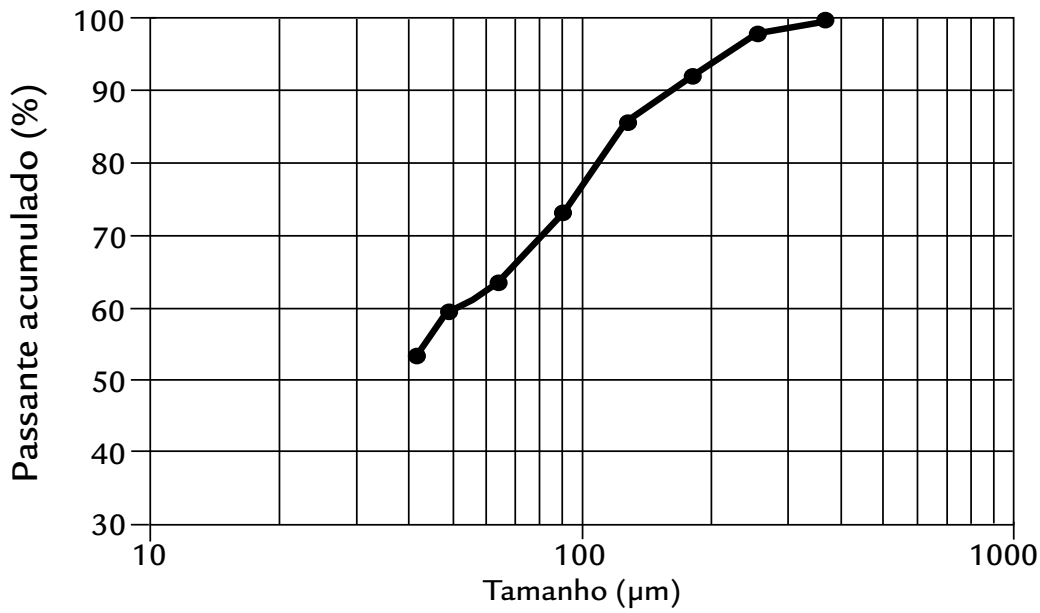

Figura 1

Análise granulométrica do minério willemítico. 
$\mathrm{pH}$ se manteve numa faixa de 10,5 a 12 . Como agente coletor e espumante, foi utilizada uma emulsão de amina, óleo diesel e MIBIC, nas proporções mássicas 1,$0 ; 0,16$ e 0,4 , respectivamente, com uma dosagem de $125 \mathrm{~g} / \mathrm{t}$. Segundo Pereira (2004), a emulsão de amina/diesel/MIBIC, nessa proporção, foi efetiva, sendo que, à medida que se aumenta a concentração da emulsão, perde-se em seletividade. Ele concluiu, também, que a concentração ótima situa-se entre 500 e $700 \mathrm{~g} / \mathrm{t}$.

Os ensaios de flotação foram realizados em célula mecânica CFB-100EEPNBA da CDC, do laboratório de

\section{Resultados e discussões}

É importante ressaltar que as concentrações utilizadas de sulfeto e amina nos testes realizados em bancada, são, respectivamente, 700 e $125 \mathrm{~g} / \mathrm{t}$, ou seja, a metade da concentração utilizada no processo industrial de flotação, no qual a recuperação conseguida é em torno de $87 \%$. A Figura 2 e a Tabela 2 mostraram que a não utilização do depressor (SD) na flotação direta de zinco levou a baixos teores, recuperação de zinco de $81,09 \%$ e

Figura 2

Teste sem depressor (SD) e sem depressor com barrilha (SDB) e performance de CMC, amido, silicato+poliacrilato (sil+poliacril), silicato de sódio, dicromato de potássio, dextrina e tanino como depressores de ganga na flotação de willemita.

Tabela 2

Recuperação de $\mathrm{Mg}(\%)$

nos testes sem depressores (SD), sem depressores com barrilha (SDB) e com depressores. tratamento de minérios do Departamento de Engenharia de Minas da UFOP, sendo que as amostras eram de $1000 \mathrm{~g}$ e que os condicionamentos nesses ensaios foram realizados com aproximadamente $30 \%$ de sólidos e com $1000 \mathrm{rpm}$, conforme o seguinte procedimento:

I. Adição de depressor, condicionamento por 2 minutos e determinação do $\mathrm{pH}$.

II. Adição de sulfeto de sódio, condicionamento por 3 minutos e determinação do $\mathrm{pH}$.

III. Adição da emulsão e condicionamento por 2 minutos.

grande recuperação de ganga dolomítica para o concentrado. Dessa forma, a introdução de depressores de dolomita reduziu os teores de óxidos de cálcio e de magnésio no concentrado, prejudiciais ao processo hidrometalúrgico do zinco.

Entre os demais depressores testados, carboximetilcelulose e amido apresentaram recuperações de $84,14 \%$ e $85,68 \%$, respectivamente, classificando-se entre os menores valores obtidos. Entretanto, em
IV. Flotação por 2 minutos com coleta de afundado e flotado.

V. Repetição da sequiência por mais duas vezes com nova adição de emulsão e sulfeto sódio, só que, desta vez, com a metade da concentração inicial desses, obtendo-se três concentrados.

Foi realizado um peneiramento a úmido seguido de análise granuloquímica com os três concentrados e rejeito do teste no qual utilizou-se tanino como depressor. Desse modo, foi possível saber em qual faixa granulométrica a willemita estava sendo perdida e, também, em qual faixa estava sendo recuperada mais dolomita. relação a teor de $\mathrm{Zn}$, no concentrado e seletividade, obtiveram-se os resultados mais expressivos, com uma menor quantidade de ganga (dolomita) no concentrado. Da mesma forma, Silva (2006), também em testes de depressão de dolomita em minério willemítico, evidenciou a seletividade da CMC. Esse depressor levou a uma menor distribuição de $\mathrm{CaO}$ no concentrado do que a cal, apesar de a última ter sido mais efetiva que a CMC.

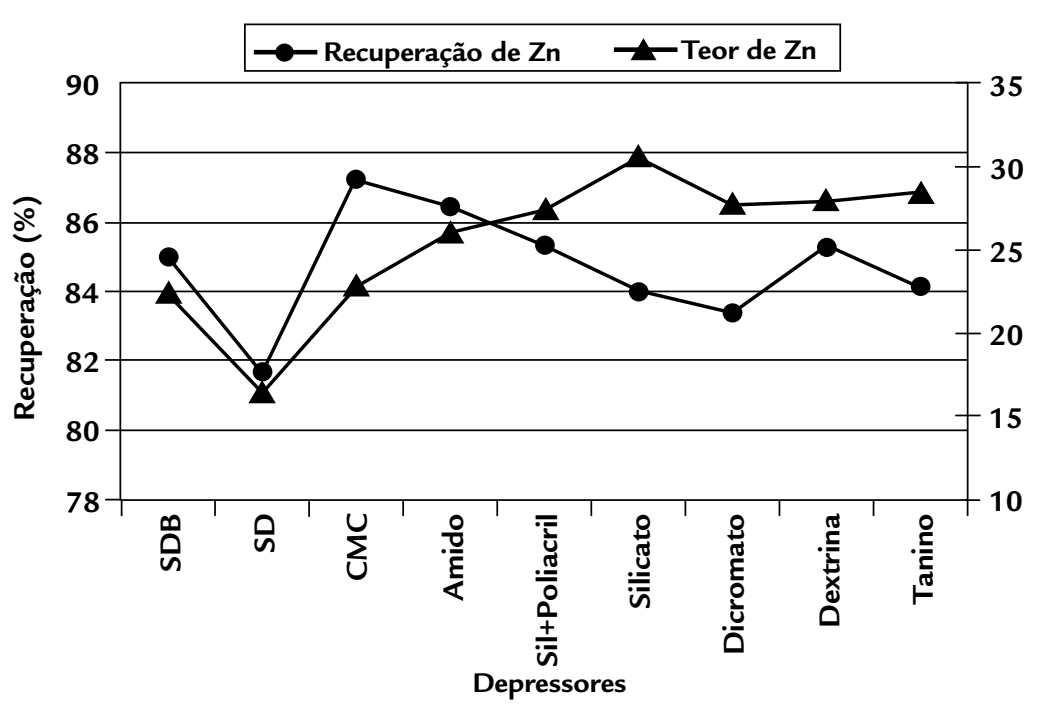

\begin{tabular}{c|c}
\hline Depressores & Recuperação de Mg no concentrado (\%) \\
\hline SD & 60.1 \\
\hline SDB & 42.8 \\
\hline CMC & 25.4 \\
\hline Tanino & 43.5 \\
\hline Dicromato & 67.2 \\
\hline Amido & 33.3 \\
\hline Silicato & 56.0 \\
\hline Silicato+poliacrilato & 41.0 \\
\hline Dextrin & 55.3 \\
\hline
\end{tabular}


Segundo a revisão da literatura feita por Baltar (1980), o dicromato foi identificado como um dos melhores depressores de ganga dolomítica, porém os testes realizados demonstraram que o dicromato de potássio apresentou uma ótima recuperação de zinco e baixos teores, com uma considerável distribuição de dolomita para o concentrado $(67,2 \%)$, evidenciando sua baixa seletividade.

Pereira (2004), a partir de testes com a calamina, observou que o poliacrilato de sódio, o qual atua aumentando e mantendo a carga negativa da dupla camada elétrica, acarretou um aumento na dispersão da lama, porém menos acentuada que a causada pelo hexametafosfato de sódio. Afirmou também que os polímeros acrilato de baixo peso molecular, quando utilizados como modificadores para minérios associados principalmente a ganga dolomítica, apresentam maior recuperação metálica, melhor seletividade, menor perda de finos e diminuição no consumo de modificadores. Nos testes realizados com a willemita, a adição de poliacrilato ao silicato de sódio significou um ganho em teor de $\mathrm{Zn}$, que aumentou em 2,5\% em relação ao teste com silicato apenas. Em relação à recuperação, o valor caiu $1,48 \%$, de $87,84 \%$ nos testes com silicato para $86,36 \%$ na presença de silicato+poliacrilato.

Nas Figuras 3 e 4, estão apresentadas, respectivamente, as análises granuloquímicas do concentrado e do rejeito da flotação realizada com o depressor tanino. Por meio da Figura 3, é possível ver, claramente, que os teores de $\mathrm{MgO}$ e $\mathrm{CaO}$ são inversamente proporcionais aos teores de $\mathrm{Zn}$ no concentrado, ou seja, sempre que há maior recuperação de willemita recupera-se menos dolomita. Desta forma, confirma-se mais uma vez a necessidade da depressão da ganga dolomítica. Os teores de $\mathrm{MgO}$ e $\mathrm{CaO}$ em granulometrias muito finas (abaixo de $38 \mu \mathrm{m}$ ) são muito altos, enquanto o de Zn é baixo. Observa-se que
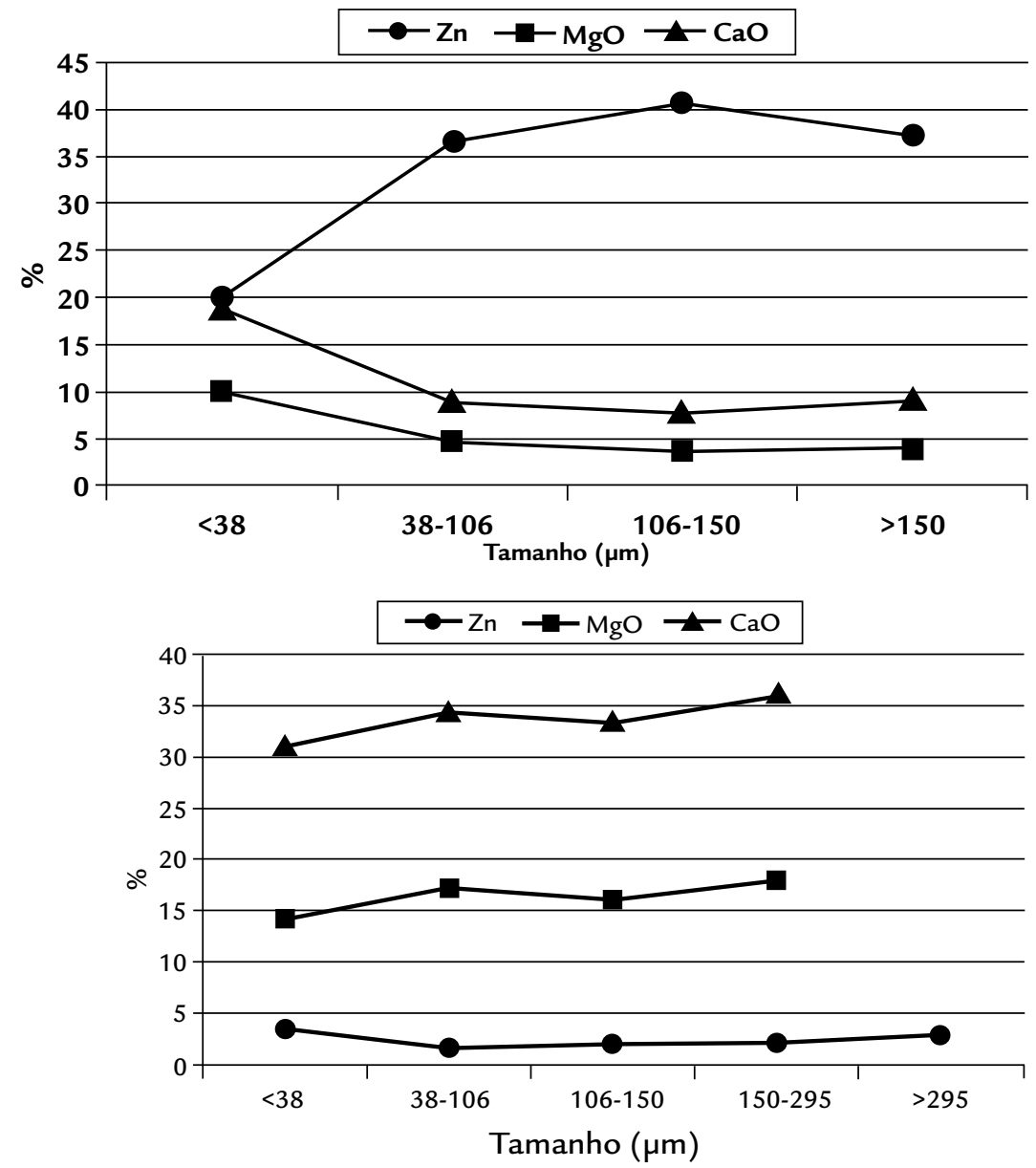

Figura 3

Análise granuloquímica do concentrado da flotação com tanino.

Figura 4

Análise granuloquímica do rejeito da flotação com tanino.

\section{Conclusões}

Essa investigação mostrou que, entre os reagentes com efeitos depressores de ganga presente em minério willemítico, o amido e o silicato de sódio associado ao a presença de dolomita cai com o aumento do tamanho das partículas, enquanto o $\mathrm{Zn}$ alcança seu teor máximo nas partículas de tamanho médio $128 \mu \mathrm{m}$.

No rejeito (Figura 4), os teores de dolomita são elevados, mas, ainda assim, há uma grande perda de Zn $(3,27 \%)$ da amostra total. As maiores perdas ocorrem no material abaixo de $38 \mu \mathrm{m}$ e acima de $295 \mu \mathrm{m}$. Provavelmente, a perda nas partículas finas deve-se à falta de seletividade nessa faixa devido ao fenômeno de "slimes coating", sendo as partículas maiores recobertas pelos finos ou lamas, causados pela presença de ganga dolomítica e ferruginosa na polpa. Já a perda nas partículas grossas, as quais representam apenas $2,7 \%$ do material, deve-se ao fato de a willemita não estar liberada nessa faixa. Devido à pequena quantidade de partículas maiores que $295 \mu \mathrm{m}$, não foi possível fazer a análise completa dessa faixa, por isso tem-se só o teor de zinco dessa amostra. poliacrilato de sódio e à carboximetilcelulose (CMC) apresentaram as maiores reduções de teores dos contaminantes, óxidos de cálcio e de magnésio, no con- centrado. Com o uso de CMC e do amido, foram obtidos concentrados mais ricos, com os maiores teores de $\mathrm{Zn}$, ou seja, esses depressores mostraram-se os mais 
seletivos. Com o dicromato de potássio, observou-se uma boa recuperação de zinco, entretanto com baixos teores e seletividade. A adição de poliacrilato ao silicato de sódio significou um ganho em teor de $\mathrm{Zn}$ para o concentrado.

\section{Referências bibliográficas}

BALTAR, C. A. M., VILLAS BOAS, R. C. Flotação de minério oxidado de zinco. Série Tecnologia Mineral, CETEM, 1980. 27 p.

BORGES, A. A. M. Influência do estado de dispersão na flotabilidade de minériooxidado de zinco. UFMG: 1993. 189 p. (Dissertação de mestrado).

GALÉRY, R. Influência do estado de dispersão na flutuabilidade do sistema willemita/dolomita. UFMG: 1985. (Dissertação de Mestrado).

GUIMARÃES, R. C., ARAUJO, A. C., PERES, A. E. C. Reagents in igneous phosphate ores flotation. Minerals Engineering, v. 18, p. 199-204, 2005.

LUVIZOTTO, G. R., LIMA, R. M. Estudo da flotabilidade da apatita, calcita e dolomita, visando a uma maior seletividade na flotação de fosfatos carbonatados. UFOP: 2003. (Relatório Final PIP).

PEREIRA, C. A. Estudo da dispersão e concentração da lama de minério oxidado de zinco. UFMG: 2004. 162p. (Tese de doutorado).

PEREIRA, C. A., PERES A. E. C. Reagents in oxidised zinc ores flotation. Minerals Engeneering, v. 18, p. 275 a 277, 2005.

PERES, A. E. C., GALERY, R. Investigação visando à redução do teor de dolomita no minério de zinco. UFMG/FCO/CMM, 1981.

SALUM, M. J. G., ARAUJO, A. C., PERES, A. E. C. The role of sodium sulphide in amine flotation of silicate zinc minerals. Minerals Engineering, v.5, p. 411-419, 1992.

SILVA, T. A. V. Estudo de reagentes na flotação de minério de zinco. UFOP: 2006. 109p. (Dissertação de mestrado).

VIANA, P. R. M. Estudo da interação entre a dolomita e reagentes de flotação. UFMG: 1981. (Dissertação de mestrado).

VIANA, P. R. M., PERES, A. E. C. Estudo da flutuabilidade da dolomita. In: ENCONTRO DO HEMISFÉRIO SUL SOBRE TECNOLOGIA MINERAL, 1. Anais... Rio de Janeiro, p. 194-203, 1982.

ZHENG, X., SMITH, R. W. Dolomite depressants in the flotation of the apatite and cellophane from dolomite. Minerals Engineering, v. 10, n. 5, p.537-545, 1997.

Artigo recebido em 17 de abril de 2010. Aprovado em 19 de julho de 2010. 